\title{
INTELIGENCIA EMOCIONAL Y LIDERAZGO AUTÉNTICO EN LOS CARGOS PÚBLICOS LOCALES ESPAÑOLES
}

\author{
D. Pedro Herrero Garcí \\ Doctorando. Universidad de Valladolid \\ herreropedro1975@gmail.com \\ Dr. Miguel Ángel Carbonero Martín \\ Universidad de Valladolid \\ Dra. Valle Flores Lucas \\ Universidad de Valladolid \\ Dr. Luis Jorge Martín Antón \\ Universidad de Valladolid
}

Fecha de Recepción: 23 Febrero 2019

Fecha de Admisión: 30 Abril 2019

\section{RESUMEN}

El desempeño de la función pública requiere de personas con un perfil personal y profesional determinado. Sin embargo, "El político medio español es un analfabeto emocional que refleja narcisismo e ignorancia" (Fernández Berrocal, en Soteras, 2015); "El nivel de inteligencia emocional en la política española es escaso" (Peñate, en Soteras, 2015). A partir de estas afirmaciones, cabría preguntarse qué esperanza podría la ciudadanía acabar depositando en la política como medio para mejorar sus vidas y transformar la sociedad y en los políticos como sus hacedores. Por ello, es imprescindible aclarar si quienes han conseguido ejercer un liderazgo político y acabar siendo elegidos para ocupar un cargo público tras ganarse la confianza de sus conciudadanos, han sido capaces de hacerlo careciendo o no de inteligencia emocional.

Siendo los ayuntamientos las instituciones más cercanas a la ciudadanía, cuyos representantes están más en contacto con los problemas de la vecindad y por tanto más expuestos al escrutinio social, cabe preguntarse si esa supuesta carencia emocional de los políticos ha podido pasar inadvertida para los votantes y si, en el caso de que así sea, dicho déficit pudiera lastrar las expectativas de que dichos representantes lleguen a ser capaces de desempeñar sus responsabilidades institucionales con alto grado de competencia desde el punto de vista moral.

Nos planteamos el estudio de la inteligencia emocional y el liderazgo auténtico de una muestra representativa de personas dedicadas al desempeño público en el ámbito de los Ayuntamientos españoles de más de 50.000 habitantes, desde la psicología positiva y sus postulados.

Palabras clave: inteligencia emocional; liderazgo auténtico; cargos públicos 


\section{INTELIGENCIA EMOCIONAL Y LIDERAZGO AUTÉNTICO EN LOS CARGOS PÚBLICOS LOCALES ESPAÑOLES}

\section{ABSTRACT}

Emotional intelligence and authentic leadership in Spanish councilors. The performance of the public function requires people with a specific personal and professional profile. However, "The average Spanish politician is an emotional illiterate that reflects narcissism and ignorance" (Fernández Berrocal, in Soteras, 2015); "The level of emotional intelligence in Spanish politics is scarce" (Peñate, in Soteras, 2015). From these statements, one could ask what hope could the citizenry end up depositing in politics as a means to improve their lives and transform society. Therefore, it is essential to clarify whether those who have managed to exercise political leadership and end up being elected to a public office after winning the trust of their fellow citizens, have been able to do so without emotional intelligence or not.

Municipalities being the institutions closest to the citizens, whose representatives are more in touch with the problems of the neighborhood and therefore more exposed to social scrutiny, it is questionable whether this alleged emotional lack of politicians has been able to pass unnoticed by voters and if, in the event that this is the case, this deficit could undermine the expectations that these representatives will be able to carry out their institutional responsibilities with a high degree of competence from the moral point of view.

We consider the study of emotional intelligence and authentic leadership of a representative sample of people dedicated to politics in the field of Spanish municipalities of more than 50,000 inhabitants, from positive psychology and its postulates.

Keywords: emotional intelligence; authentic leadership; politicians

\section{ANTECEDENTES}

El Centro de Investigaciones Sociológicas (CIS) refleja en su barómetro de febrero de 2018 que "Ios políticos en general, los partidos políticos y la política", en su conjunto, son concebidos como uno de los "tres principales problemas que existen actualmente en España" por parte del $24,2 \%$ de la ciudadanía (CIS, 2018). No es este, sin embargo, un dato que pueda considerarse coyuntural teniendo en cuenta que estos mismos estudios se vienen realizando al menos desde 1985, reflejándose en ellos este problema de forma más o menos intensa pero permanente a lo largo de los años, por lo que bien podría considerarse, en todo caso, un problema "estructural".

Esta percepción negativa de una parte de la sociedad hacia las personas dedicadas a la política no puede considerarse, sin embargo, exclusiva de la época actual o moderna. El ejercicio de la política se ha vinculado, a lo largo de la historia, con variedad de actitudes y comportamientos perniciosos y censurables como la mentira, la manipulación, la corrupción, etc. En este sentido, Jordá y Catalá (2017) atribuyen al diplomático español Diego de Saavedra Fajardo (1584-1654) la frase: "Todo el estudio de los políticos se emplea en cubrirle el rostro a la mentira para que parezca verdad, disimulando el engaño y disfrazando los designios".

Igualmente, estos autores imputan al filósofo inglés Aldoux Huxley (1894-1963) esta otra que dejó para la posteridad: "Cuanto más siniestros son los deseos de un político, más pomposa, en general, se vuelve la nobleza de su lenguaje", mientras que Laercio, Riaño y Sanz (1962, p. 133) imputan a Antístenes (Filósofo griego fundador de la Escuela cínica) la sentencia: "Las ciudades se pierden cuando no pueden discernir a los viles de los honestos", por lo que cabe preguntarse: ¿Hasta qué punto tiene fundamento esta percepción tan extendida en el tiempo?, ¿Es un mito, 0 tiene visos de irrefutabilidad? Y, de forma aún más trascendente: ¿Cómo puede distinguir la ciudadanía a los viles de los honestos?

Si tenemos en cuenta que, en democracia, las personas que acaban siendo elegidas para ocupar un cargo público lo han sido tras haberse ganado previamente la confianza de sus iguales (seguidores, compañeros y simpatizantes en sus formaciones políticas) y más tarde de sus conciu- 
dadanos (especialmente en el ámbito municipal, que es aquel en que más exposición hay al escrutinio público), ¿En efecto habrán podido ser capaces de conseguirlo careciendo o con muy bajo nivel de inteligencia emocional (IE)?, ¿Cursa esa IE con el liderazgo que se supone están capacitados para ejercer y de hecho a menudo ejercen?

Cada uno desde su enfoque, los distintos modelos explicativos de Inteligencia Emocional hacen referencia a variables como la empatía (Goleman, 1995), el autocontrol (Vallés y Vallés, 1999), las habilidades sociales (Elias, Tobias y Friedlander, 1999), la asertividad y la adaptabilidad (Bar-On, 1997), la motivación positiva Martineaud y Engelhartn, 1996) o la dirección emocional (Salovey y Mayer, 1990). Todas esas corrientes apuntan cualidades que caben entenderse como necesarias, si no imprescindibles para el ejercicio de la actividad política, habida cuenta de que ésta tiene un marcado carácter social, especialmente en el ámbito local, más ligado al conocimiento y la interacción personal directa entre vecinos.

La obra titulada "Política y emoción" de Bisquerra (2016), que hace referencia a la distancia emocional que separa a representantes y representados, sirve como estímulo inicial para acercarse al estudio de este fenómeno. Acerca de la relación específica entre la Inteligencia Emocional y liderazgo en personas dedicadas a la política, cabe referir algunas aproximaciones, entre las que destaca la de Goleman (2014) vinculando estrechamente ambas variables. Por su parte, García Nuñez del Arco (2006) también relacionó el liderazgo con la Psicología política y concretamente con la conciencia política.

En ese mismo sentido, Nussbaum (2014) analiza la inteligencia emocional de grandes líderes políticos como Mahatma Gandhi, Jawaharlal Nehru, Abraham Lincoln y Martin Luther King Jr. y su trascendencia en la formación de movimientos y cambios sociales. Un paso más allá, van Marcus, Neuman y Mackuenn (2007) al vincular las emociones con el juicio político en escenarios no electorales y otorgar prevalencia a los componentes emocionales sobre los racionales en la formación de opinión y tendencia política.

mos del análisis de los modelos de Inteligencia Emocional elaborado por García-Fernández y Giménez-Mas (2010), eligiendo el Modelo "de habilidades" propuesto por Mayer, Salovey y Caruso (2000), que no incluye factores de personalidad y que hace hincapié en la importancia de estos cinco aspectos:

La percepción emocional (de uno mismo y los demás).

La facilitación emocional del pensamiento: la capacidad para usar las emociones al relacionar sensaciones, utilizar el razonamiento y dirigir la atención.

La comprensión emocional, enfocada a la resolución de problemas.

La dirección emocional: la comprensión de las implicaciones de los actos sociales en las emociones.

La regulación reflexiva de las emociones: para el crecimiento personal.

En lo que respecta al liderazgo, Daft (2006) lo concibe genéricamente como una capacidad de influencia sobre otras personas para generar un cambio y llegar a resultados reales que reflejen los propósitos compartidos. Un concepto que este autor considera que se ha ido desarrollando a lo largo de la historia en sucesivas teorías. A finales del siglo XX, con el auge de la psicología positiva, primero surgió el interés por la autorrealización de las personas líderes y posteriormente también por la de quienes trabajan con ellas, comenzándose a hablar de un "liderazgo auténtico" (LA). Este enfoque ha proliferado en los últimos años, según Moriano, Molero y Lévy Mangin (2011, p. 336) a consecuencia, en buena medida, de los efectos de la gran crisis económica en una amplia parte del mundo, considerando que "el comportamiento decepcionante de algunos líderes políticos". De esa forma, el LA aspira a ser concebida como antítesis de los liderazgos que pueden ampa- 


\section{INTELIGENCIA EMOCIONAL Y LIDERAZGO AUTÉNTICO EN LOS CARGOS PÚBLICOS LOCALES ESPAÑOLES}

rar la falsedad, sirviendo así para mejorar la cultura de las organizaciones, la motivación, el compromiso y la satisfacción laboral (Jensen y Luthans, 2006).

A este respecto, el concepto de LA contempla precisamente, en buena medida, ese significado de autenticidad. No se trata de ningún novedoso planteamiento, sino que resulta derivar más bien de un valor clásico, ahora rescatado y puesto de relieve, pero ya apreciado en la antigua Grecia (Avolio y Gardner, 2005).

Avolio y Luthans (en Cameron et al., 2003) fueron de los primeros investigadores en plantear este modelo de liderazgo basado en la autenticidad, denominado "Liderazgo Auténtico". Según ellos, las personas que ejercen el liderazgo con autenticidad no lo hacen en el sentido de limitarse a actuar siguiendo sus impulsos o intuiciones de forma despreocupada, sino que se esmeran en analizarse tanto a sí mismas como a su entorno y a las personas con las que colaboran e interactúan para detectar y potenciar sus talentos, actúan con transparencia y lo hacen de forma coherente. No miran sólo por sus intereses, por tanto, sino también por quienes las rodean, por lo tanto, no son líderes individualistas.

En este sentido, Moriano, Molero y Lévy Mangin (2011, p. 337) se hacen eco de la definición que realizan Walumbwa et al, (2008, p. 89), siendo la más citada en las investigaciones sobre el LA:

"(...) un patrón de conducta que promueve y se inspira tanto en las capacidades psicológicas positivas como en un clima ético positivo, para fomentar una mayor conciencia de uno mismo, una moral internalizada, un procesamiento de la información equilibrado y transparencia en las relaciones entre el líder y los seguidores".

Estos autores destacan la importancia de la capacidad de las personas que ejercen LA para cultivar la identificación personal y social con sus proyectos, constituyendo lo que denominan un "constructo multidimensional de orden superior". Podría decirse, por tanto, que estas personas no aspiran sólo a ejercer, mantener o reforzar su liderazgo, sino a hacerlo por la vía de la seducción 0 el convencimiento:

La "perspectiva moral internalizada", que está vinculada a los niveles más altos del desarrollo moral de la persona que lidera y se caracteriza por comportamientos guiados por principios morales y valores personales y no por impulsos o por presiones externas. De esta forma, cabe esperar de las personas con gran perspectiva moral internalizada que cuando se enfrenten a grandes desafíos éticos piensen bajo una perspectiva ética más amplia (Werhane, 1999).

La "autoconciencia", que tiene que ver con el nivel de autoconocimiento de la persona que lidera en su capacidad de influencia en otras personas (Campbell, Trapnell, Heine, Katz, Lavallee y Lehman, 1996). A mayor nivel de autoconciencia, mejor aprehensión de los valores, ideales y creencias, así como fortalezas y debilidades por parte de quien lidera y más capacidad para liderar y ampliar el número de personas que la siguen.

La "transparencia relacional", que concierne a los intercambios de información y revelaciones de la persona que lidera con las demás, incluyendo sus verdaderos pensamientos y sentimientos. La transparencia relacional está marcada por la apertura, la responsabilidad y la honestidad entre quienes lideran y las personas que las siguen (Avolio, Luthans y Ryan, 2006), promoviendo intercambios sociales más claros donde está claro lo que cada uno espera de la relación.

El "procesamiento equilibrado", que es la capacidad para analizar de forma objetiva información relevante antes de llegar a una conclusión 0 decisión. Las personas que lideran y demuestran un procesamiento equilibrado solicitan con frecuencia las opiniones de quienes las siguen, están dispuestas a debatir sobre sus propias posiciones 0 creencias antes de tomar una decisión e invierten tiempo analizando las causas de los problemas y sus efectos, contribuyendo así a discernir el locus de control ético interno 0 externo (Gardner et al., 2005). 
La persona que ejerce un LA se caracteriza, según Eilam y Shamir (2005), por lo siguiente:

No falsifica su liderazgo ni lo disfraza para aparentar, por lo que su comportamiento es genuino.

No asume su responsabilidad o su rol de liderazgo sólo por detentarlo, por vanidad o como medio para obtener recompensas personales, sino para desarrollar un proyecto, desde una convicción y para defender una causa o cumplir una misión. Es, por tanto, un liderazgo verdadero o eudaimónico (dicho en términos aristotélicos).

Es un liderazgo original, es decir, no copia ni imita a otras personas. Aunque sus valores y convicciones no puedan ser totalmente originales, éstos han sido adaptados y personalizados mediante sus aprendizajes vitales y reflexiones.

Se conduce con integridad: sus acciones responden a la aplicación de sus valores y convicciones y no persiguen la popularidad, complacer a la audiencia 0 alcanzar un objetivo particular, lo que les permite actuar con un alto nivel de transparencia.

Por todo ello, consideramos que el LA es el tipo de liderazgo más adecuado para el propósito del estudio, al estar ligado a la política y los políticos.

\section{OBJETIVOS DE LA INVESTIGACIÓN}

Medir la IE y el LA de los políticos podría servir para confirmar o desmentir el fundamento de las aseveraciones que se apuntaban al comienzo, respecto a las supuestas carencias de los cargos públicos, así como el efecto de ese déficit en el ejercicio de sus funciones y por tanto en sus decisiones hacia el conjunto de la ciudadanía. Los objetivos de la investigación, por tanto, son tres:

Medir el nivel de Inteligencia Emocional de los cargos públicos locales en España.

Medir el nivel de Liderazgo auténtico de los cargos públicos locales en España.

Analizar la relación entre la IE y el LA de dichos cargos públicos.

\section{PARTICIPANTES}

Aunque ciñamos la muestra al ámbito político de las entidades locales, hay que tener en cuenta que en España suman un total de 7.364, entre ayuntamientos, diputaciones provinciales, consejos insulares y cabildos insulares, que están dispersas por toda la geografía nacional y en los que prestan sus servicios más de 64.000 ediles. Esta circunstancia obliga a restringir la muestra, de forma que pueda ser lo más representativa posible. Por ello, hemos decidido considerar sólo aqueIlas localidades que se correspondan con poblaciones superiores a los 50.000 habitantes, es decir, un total de 145 .

\section{METODOLOGÍA E INSTRUMENTOS}

Al tratarse de una investigación de carácter descriptivo y correlacional, se seleccionará una muestra representativa de la población que se pretende estudiar (cargos públicos de las mencionadas entidades locales con más de 50.000 habitantes) y se contactará con ellos mediante correo electrónico, enviándoles un cuestionario digital que para la medida de las variables objeto de estudio. Dicho cuestionario integrará los dos instrumentos elegidos para realizar la investigación, que son los siguientes:

Para la medición de la Inteligencia Emocional utilizaremos la Wong-Law Emotional Intelligence Scale (WLEIS) de 2002. Este instrumento tiene un formato de autoinforme compuesto por 16 elementos que abarcan cuatro factores: la evaluación de las propias emociones (percepción intrapersonal), la evaluación de las emociones de los demás (percepción interpersonal), el uso de las emociones o asimilación, y la regulación de las emociones.

Por otro lado, para la medición del Liderazgo Auténtico emplearemos el Authentic Leadership 


\section{INTELIGENCIA EMOCIONAL Y LIDERAZGO AUTÉNTICO EN LOS CARGOS PÚBLICOS LOCALES ESPAÑOLES}

Questionaire ALQ (Avolio et al., 2008) en su versión para la persona que lidera (la otra está destinada a las personas seguidoras), que está compuesta por 16 ítems en un formato de respuesta de frecuencia mediante escala Likert de 5 opciones (de 0 a 4) que contrapone versiones y contempla cuatro factores: la conciencia de sí mismo (en referencia al conocimiento sobre las propias capacidades y limitaciones y las ajenas, así como a la forma en que las propias conductas afectan a los demás), la transparencia (en relación al comportamiento natural que genera confianza en el entorno de las personas que rodean a quien lidera), el procesamiento equilibrado (relativo al análisis pormenorizado de la información y del contexto antes de tomar decisiones, consultando opiniones) y la moral internalizada (vinculada a la coherencia entre la conducta y los valores o principios frente a presiones internas o externas). La versión española de este cuestionario corresponde a Moriano, Molero y Lévy Mangin (2011).

Ambos instrumentos están validados en España Posteriormente. Los datos obtenidos se someterán al tratamiento estadístico pertinente para la obtención de resultados y su posterior interpretación.

\section{RESULTADOS, DISCUSIÓN Y CONCLUSIONES}

En este momento del proceso de investigación, el trabajo está siendo dedicado al diseño del cuestionario, en el marco de la elaboración de una tesis doctoral. Esperamos que los resultados no sólo confirmen la correlación entre el LA y la IE, de forma que no sea posible ejercer el primero sin un adecuado nivel de la segunda, sino que, además, pueda contrastarse con otras variables independientes como la experiencia política, de forma que el ejercicio de la política repercuta en un mayor LA como fruto del aprendizaje.

La discusión de los resultados puede dar pie a la necesidad de comparación con otro los que se obtendrían en otro tipo de ámbitos profesionales, de modo que se pudiera contrastar si en efecto quienes se dedican a la política tienen niveles de IE y LA superiores a los de otros sectores o por el contrario si este colectivo representa una simple muestra de ellos, como se supone.

Entendemos que las conclusiones de este estudio pueden resultar de gran utilidad en el campo de la formación, teniendo en cuenta que actualmente los cargos públicos locales disponen de acceso a programas de mejora impulsados por las propias Administraciones Públicas, cada vez más concienciadas con la necesidad de mejorar las capacidades de los servidores públicos, especialmente en el ámbito municipal.

\section{REFERENCIA BIBLIOGRÁFICA}

Avolio, B. J. y Luthans, F. (2003). Authentic leadership: A positive development approach. En Cameron, K.S., Dutton, J. E. y Quinn, R.E. (Eds.) Positive organizational scholarship: Foundations of a new discipline (pp. 241-261), San Francisco, Berrett-Koehler.

Avolio, B.J., Gardner, W.L., Peterson, S.J., Walumbwa, F.O. y Wernsing, T.S. (2008). Authentic leadership: Development and validation of a theory-based measure. Journal of management, 34 (1), pp. 89-126.

Avolio, B. J., Luthans, F., y Ryan, C. (2006). The high impact leader. New York: McGraw Hill.

Bar-On, R. (1997). The Emotional Quotient inventory (EQ-I): Technical Manual, Toronto, Canadá: Multi-Health Systems.

Bisquerra, R. (2016). Política y emoción. Madrid, España. Pirámide.

Campbell, J. D., Trapnell, P. D., Heine, S. J., Katz, I. M., Lavallee, L. F., y Lehman, D. R. (1996). Selfconcept clarity: Measurement, personality correlates, and cultural boundaries. Journal of personality and social psychology, 70 (1), p. 141. 
Centro de Investigaciones Sociológicas (2018). Barómetro de febrero. http://www.cis.es/cis/export/sites/default/-Archivos/Indicadores/ documentos html/TresProblemas.html.

Daft, R. L. (2006). La Experiencia del Liderazgo. México: CENGAGE Learning.

Eilam, G. y Shamir, B. (2005). What's your story? A life-stories approach to authentic leadership development. The Leadership Quarterly, 16, pp. 395-417

Elias, M., Tobias, S. y Friedlander, B. (1999). Educar con inteligencia emocional, Barcelona. Plaza y Janés.

García-Fernández, M., y Giménez-Mas, S. I. (2010). La inteligencia emocional y sus

principales modelos: propuesta de un modelo integrador. Cuadernos del

profesorado 3(6), pp. 43-52.

García Núñez del Arco, C. (2006). Conciencia política y liderazgo. Liberabit, 12 (12), pp. 93-100.

Goleman, D. (1995). Emotional intelligence. New York: Bantam.

Goleman, D. (2014). Liderazgo. El poder de la inteligencia emocional. Barcelona, España. Ediciones B.

Jensen, S. M. y Luthans, F. (2006). Relationship between Entrepreneurs' Psychological Capital and Their Authentic Leadership. Journal of Managerial Issues, 18 (2), pp. 254-273.

Jordá, V. y Catalá, X. (2017). Provervia.net. Gandía, España. Novixar. http://www.proverbia.net/citastema.asp?tematica $=58$

Laercio, D., Riaño, J.M. y Sanz, J.0. (1962). Vidas de los filósofos ilustres. Iberia.

Marcus, G., Neuman, R., y Mackuenn, M. (2007). Inteligencia afectiva y juicio político. Primera parte. Sociológica (México), 22 (63), pp. 253-266.

Martineaud, S. y Engelhart, D. (1996). El Test de inteligencia emocional. Barcelona: Martínez Roca.

Mayer, J. D., Salovey, P., y Caruso, D. (2000). Models of emotional intelligence. 2, pp. 396-420. Cambridge University Press, Reino Unido. Handbook of intelligence.

Moriano, J.A., Molero, F. y Lévy Mangin, J. P. (2011). Liderazgo auténtico. Concepto y validación del cuestionario ALQ en España. Psicotema, 23 (2), pp. 336-341.

Nussbaum, M. C. (2014). Emociones políticas. Barcelona, España: Paidós.

Salovey, P., y Mayer, J.D. (1990). Emotional intelligence. Imagination, Cognition, and Personality, 9 (3), pp. 185-211.

Soteras, A. (2015). www.efesalud.com Recuperado de: https://www.efesalud.com/los-politicos-suspenden-en-inteligencia-emocional-salvo-los-emergentes/

Vallés, A. y Vallés, C. (1999). Desarrollando la inteligencia emocional. Madrid: EOS.

Walumbwa, F.O., Avolio, B.J., Gardner, W.L., Wernsing, T.S. y Peterson, S.J. (2008). Authentic leadership: Development and validation of a theory-based measure. Journal of management, 34 (1), pp. 89-126.

Werhane, P. H. (1999). Moral imagination and management decision-making. Oxford University Press, USA. 
